\title{
Nield-Kuznetsov Functions: Current Advances and New Results
}

\author{
M.H. Hamdan \\ hamdan@unb.ca \\ Department of Mathematics and Statistics \\ University of New Brunswick \\ Saint John, N.B., E2L 4L5 \\ Canada \\ S. Jayyousi Dajani \\ sdajani@mx.lakeforest.edu \\ Department of Mathematics and Computer Science \\ Lake Forest College \\ Lake Forest, IL 60045 \\ USA \\ M.S. Abu Zaytoon \\ mohammad.abuzaytoon@uop.edu.jo \\ Department of Mathematics \\ University of Petra \\ Amman \\ Jordan
}

Received: March 27, 2021. Received: August 26, 2021. Accepted: September 18, 2021. Published: September $20,2021$.

\begin{abstract}
In this article, we discuss a class of functions known as the Nield-Kuznetsov functions, introduced over the past decade. These functions arise in the solutions to inhomogeneous Airy's and Weber's equations. Derivations of these functions are provided, together with their methods of computations.
\end{abstract}

Key-Words: - - Nield-Kuznetsov, Weber, Airy, Inhomogeneous

\section{INTRODUCTION}

Many interesting equations of mathematical physics have their roots in the nineteenth century, and arose then due to their various applications in physics. Their solutions have been expressed in the forms of integral functions which gave rise to various special functions that we know today.

Two examples of the said equations are Airy's and Weber's differential equations. Both equations continue to receive considerable attention in the literature ( $c f .[1,2,3,4]$ and the references therein) due to their various practical applications and theoretical implications. In addition to its direct applicability in the study of optics, fluid flow and electromagnetism, many other differential equations in mathematical physics can be reduced to Airy's or Weber's equations by an appropriate change of variables. Their theoretical implications are found in the introduction of other functions in seeking their solutions, and infinite series analysis of their solutions and the functions that are expressible in terms of Airy's functions and Weber's functions ( $c f$. $[5,6,7]$ and the references therein). In addition, 
these functions contribute to the creation of knowledge in their extensions to large arguments and complex domains of validity, [8], and generalizations of these functions whenever possible and necessary, [9].

Computationally, Airy's, Weber's, and other related special functions continue to represent a challenge and give rise to the need for efficient computational algorithms, (cf. $[10,11,12]$ and the references therein). In fact, a large number of scientists have devoted the better parts of their careers to provide us with the State-of-the-Art computational methodologies of these special functions ( $c f .[13,14,15,16,17]$ and the references therein).

Although Airy's and Weber's equations started out in their homogeneous forms, it is now evident that many applications require these equations in their more general, inhomogeneous forms with forcing functions that can be either constant of variable. For instance, Airy's inhomogeneous equation proved to be a valuable tool in the analysis of flow over porous layers in the presence of a transition layer, wherein Nield and Kuznetsov [18] showed that Brinkman's equation, which governs the flow in the variable permeability transition layer, can be reduced to Airy's equation. Abu Zaytoon et.al. $[19,20]$ provided models of variable permeability porous layers that resulted in reducing the governing equations to the generalized Airy's equation and Weber's equation.

Applications of the inhomogeneous Airy's and Weber's equations underscore the fundamental importance of solving their initial and boundary value problems, and providing the necessary computational methodologies to evaluate their integral functions. To this end, Nield and Kuznetsov [18] found it convenient to define a new integral function in terms of Airy's functions and their integrals. This provided a methodology of handling the inhomogeneous Airy's equation with a constant forcing function.

In systems theory, Airy's equation and functions arise in connection with Wigner distribution and the establishment of connections between chaotic classical systems and their quantum correspondents (cf. [4] and the references therein). Furthermore, their applications in the theory of elasticity, and in the analysis of Schrodinger equation are welldocumented, so are their uses in evaluation of the Franck-Condon factors that arise in connection with the Franck-Condon principle. This principle is used to describe transitions between two electronic states of a molecule, [4]. In cases where variable forcing functions arise in system theory applications, the introduced Nield-Kuznetsov functions will become a viable alternative. At this stage, however, only a few applications that rely on the Nield-Kuznetsov functions have been discussed in the literature.

Properties of this newly-introduced integral function have been studied by Hamdan and Kamel, [21] and [22], who also introduced an integral function to express the particular solution of Airy's inhomogeneous equation with a variable forcing function. Integral functions suitable for expressing particular solutions of the generalized Airy's equation and the generalized Weber's equation were introduced by Abu Zaytoon et.al. [19], [20], and Alzahrani et.al. [23], and analyzed extensively by the same authors $(c f .[24,25,6,27,28,29,30,31])$.

The current work stems from our belief that knowledge of the equations of mathematical physics, and their associated solutions in the forms of integral and special functions, is an important aspect of modern research. These functions represent our mathematical treasure, and their computations are an integral part of our methodologies to expand this knowledge. While it might be true that problems involving Airy's equation or Weber equation, with or without a forcing function, can be solved numerically or, in some cases, using power series solutions or even Homotpy Perturbation Methods, there are reasons as to why one needs functions such as Airy's function, Scorer functions, parabolic cylindrical functions and the Nield-Kuznetsov functions. These reasons include the fact that knowing the functional form of the solution, its functional components, how the solution depends on these functions, the behaviours of these functions as parameters change, 
representations of these functions, their interdependence, and the relationships these functions have with other functions offers us more confidence in our solutions and our robust methodologies. Furthermore, they represent an excellent tool in the transfer of knowledge both inter-discipline and intra-discipline. Suffice it to say that had Sir G.B. Airy obtained an answer to his question than a solution in terms of what we now know as Airy's functions, we would be discussing a different topic of interest at present.

Connections that Airy's functions and the NieldKuznetsov functions have with other functions, such as Bessel functions, offers us further insights into behaviours and methods of evaluation of these functions. Furthermore, extensions of applications of these functions, and the design of methodologies that combine solution methodologies obtained using these functions and other techniques, such as numerical approaches, is quite possible. It will not come as a surprise to us if Airy's functions and the Nield-Kuznetsov functions find their way into grid generation techniques, finite difference methods, or the finite element method. The work of Himeur et.al. [32] discusses, and provides an excellent formulation of a higher order triangular plate finite element using Airy's functions.

While we feel this is just the tip of the iceberg when it comes to advancing knowledge from homogeneous to inhomogeneous equations, and that there are many other equations to consider, our goal here is to provide this most up to date and comprehensive knowledge of six functions that have been developed over the past decade. Three classes of the Nield-Kuznetsov functions are considered (the Standard, the Generalized, and the Parametric Nield-Kuznetsov functions), each of which is of two kinds: the first and second kinds. They cover the two cases of inhomogeneities: a constant forcing function (Nield-Kuznetsov functions of the first kind) and variable forcing functions (NieldKuznetsov functions of the second kind).

In this work, we provide details of their derivations, and their ascending series representations that are suitable for efficient computations. Some sample computations are provided for illustration.

\section{THE STANDARD NIELD-KUZNETSOV FUNCTIONS}

A. Initial Value Problem

Consider the inhomogeneous Airy's ordinary differential equation (ODE):

$\frac{d^{2} y}{d x^{2}}-x y=f(x)$

subject to the initial conditions

$y(0)=\alpha$

(2)

$\frac{d y}{d x}(0)=\beta$

where $\alpha$ and $\beta$ are known values.

Solutions to (1) depend on the forcing, $f(x)$, which gives rise to the following four cases.

\section{A.1. The Case of $f(x) \equiv 0$.}

In this case, general solution to (1) is given by

$y=a_{1} A_{i}(x)+a_{2} B_{i}(x)$

where $a_{1}$ and $a_{2}$ are arbitrary constants, and the functions $A_{i}(x)$ and $B_{i}(x)$ are two linearly independent functions known as Airy's homogeneous functions of the first and second kind, respectively, and are defined by the following integrals, [4]:

$A_{i}(x)=\frac{1}{\pi} \int_{0}^{\infty} \cos \left(x t+\frac{t^{3}}{3}\right) d t$

$B_{i}(x)=\frac{1}{\pi} \int_{0}^{\infty}\left[\sin \left(x t+\frac{t^{3}}{3}\right)+\exp \left(x t-\frac{t^{3}}{3}\right)\right] d t$

The non-zero Wronskian of $A_{i}(x)$ and $B_{i}(x)$ is given by, [4]:

$W\left(A_{i}(x), B_{i}(x)\right)=$ 
$A_{i}(x) \frac{d B_{i}(x)}{d x}-B_{i}(x) \frac{d A_{i}(x)}{d x}=\frac{1}{\pi}$

Solution (4) satisfying the initial conditions (2) and (3) takes the form

$$
\begin{aligned}
& y=\pi\left\{\frac{(3)^{\frac{1}{6}} \alpha}{\Gamma\left(\frac{1}{3}\right)}-\frac{\beta}{(3)^{\frac{1}{6}} \Gamma\left(\frac{2}{3}\right)}\right\} A_{i}(x)+ \\
& \pi\left\{\frac{\alpha}{(3)^{\frac{1}{3}} \Gamma\left(\frac{1}{3}\right)}+\frac{\beta}{(3)^{\frac{2}{3}} \Gamma\left(\frac{2}{3}\right)}\right\} B_{i}(x)
\end{aligned}
$$

where $\Gamma($.$) is the Gamma function.$

\section{A.2 The Case of $f(x)=\mp \frac{1}{\pi}$.}

In these cases, general solution is given by a linear combination of Airy's functions and Scorer functions, [4], [6], as follows.

For $f(x)=-\frac{1}{\pi}$, general solution of (1) is given by

$y=b_{1} A_{i}(x)+b_{2} B_{i}(x)+G_{i}(x)$

(9)

where $b_{1}$ and $b_{2}$ are arbitrary constants, and

$G_{i}(x)=\frac{1}{\pi} \int_{0}^{\infty} \sin \left(x t+\frac{t^{3}}{3}\right) d t$

Solution to the initial value problem (1), (2), (3), in this case takes the form

$y=\pi\left\{\frac{(3)^{\frac{1}{6}} \alpha}{\Gamma\left(\frac{1}{3}\right)}-\frac{\beta}{(3)^{\frac{1}{6}} \Gamma\left(\frac{2}{3}\right)}\right\} A_{i}(x)+\pi\left\{\frac{\alpha}{(3)^{\frac{1}{3}} \Gamma\left(\frac{1}{3}\right)}+\right.$

$\left.\frac{\beta}{(3)^{\frac{2}{3}} \Gamma\left(\frac{2}{3}\right)}-\frac{1}{3 \Gamma\left(\frac{1}{3}\right) \Gamma\left(\frac{2}{3}\right)}\right\} B_{i}(x)+G_{i}(x)$

(11)

For $f(x)=\frac{1}{\pi}$, general solution of $(1)$ is given by

$y=c_{1} A_{i}(x)+c_{2} B_{i}(x)+H_{i}(x)$

(12)

where $c_{1}$ and $c_{2}$ are arbitrary constants, and
$H_{i}(x)=\frac{1}{\pi} \int_{0}^{\infty} \exp \left(x t-\frac{t^{3}}{3}\right) d t$

Solution to the initial value problem (1), (2), (3), in this case takes the form

$$
\begin{aligned}
& y=\pi\left\{\frac{(3)^{\frac{1}{6}} \alpha}{\Gamma\left(\frac{1}{3}\right)}-\frac{\beta}{(3)^{\frac{1}{6}} \Gamma\left(\frac{2}{3}\right)}\right\} A_{i}(x)+\pi\left\{\frac{\alpha}{(3)^{\frac{1}{3}} \Gamma\left(\frac{1}{3}\right)}+\right. \\
& \left.\frac{\beta}{(3)^{\frac{2}{3}} \Gamma\left(\frac{2}{3}\right)}-\frac{4}{(3)^{\frac{2}{3}} \Gamma\left(\frac{1}{3}\right) \Gamma\left(\frac{2}{3}\right)}\right\} B_{i}(x)+H_{i}(x)
\end{aligned}
$$

In applying the initial conditions (2) and (3) to obtain values of the arbitrary constants, we relied on values of the integral functions $A_{i}(x), B_{i}(x)$, $G_{i}(x), H_{i}(x)$ and their first derivatives at $x=0$, reported in [4]. These values are listed Tables 1(a) and $1(\mathrm{~b})$, below.

\begin{tabular}{|c|}
$\begin{array}{l}\text { Values of Airy's and Scorer } \\
\text { functions at } \boldsymbol{x}=\mathbf{0}\end{array}$ \\
\hline$A_{i}(0)=\sqrt{3} G_{i}(0)=\frac{\sqrt{3}}{(3)^{\frac{7}{6}} \Gamma\left(\frac{2}{3}\right)}$ \\
$B_{i}(0)=3 G_{i}(0)=\frac{3}{(3)^{\frac{7}{6}} \Gamma\left(\frac{2}{3}\right)}$ \\
$G_{i}(0)=\frac{A_{i}(0)}{\sqrt{3}}=\frac{1}{(3)^{\frac{7}{6}} \Gamma\left(\frac{2}{3}\right)}$ \\
$H_{i}(0)=\frac{2 B_{i}(0)}{3}=\frac{2}{(3)^{\frac{7}{6}} \Gamma\left(\frac{2}{3}\right)}$ \\
\hline
\end{tabular}

Table 1(a)

Values of the first derivatives of Airy's and Scorer functions at $\boldsymbol{x}=\mathbf{0}$

$$
\frac{d A_{i}(0)}{d x}=-\sqrt{3} \frac{d G_{i}(0)}{d x}=\frac{-\sqrt{3}}{(3)^{\frac{5}{6}} \Gamma\left(\frac{1}{3}\right)}
$$




\begin{tabular}{|c|}
\hline$\frac{d B_{i}(0)}{d x}=3 \frac{d G_{i}(0)}{d x}=\frac{3}{(3)^{\frac{5}{6}} \Gamma\left(\frac{1}{3}\right)}$ \\
$\frac{d G_{i}(0)}{d x}=-\frac{1}{\sqrt{3}} \frac{d A_{i}(0)}{d x}=\frac{1}{(3)^{\frac{5}{6}} \Gamma\left(\frac{1}{3}\right)}$ \\
$\frac{d H_{i}(0)}{d x}=\frac{2}{3} \frac{d B_{i}(0)}{d x}=\frac{2}{(3)^{\frac{5}{6}} \Gamma\left(\frac{1}{3}\right)}$ \\
\hline
\end{tabular}

Table 1(b)

\section{A.3 The Case of $f(x)=R=$ constant}

In Case 2, above, the Scorer functions furnish the particular solution to inhomogeneous Airy's ODE for the special values $f(x)=\mp \frac{1}{\pi}$. If $f(x)=R$ is any constant or variable function of $x$, the Scorer functions do not directly render the needed particular solution.

When $f(x)=R$, a particular solution to ODE (1) is obtained using the method of variation of parameters to obtain the following general solution:

$y=d_{1} A_{i}(x)+d_{2} B_{i}(x)-\pi R N_{i}(x)$

where $d_{1}$ and $d_{2}$ are arbitrary constants, and

$N_{i}(x)=A_{i}(x) \int_{0}^{x} B_{i}(t) d t-B_{i}(x) \int_{0}^{x} A_{i}(t) d t$

The integral function $N_{i}(x)$ is called the Standard Nield-Kuznetsov Function of the First Kind. Nield and Kuznetsov, [18], introduced (16) in their study of the effect of transition layer in the flow over porous layers. Hamdan and Kamel [21] established the following relationships of $N_{i}(x)$ to Airy's and Scorer's functions:

$N_{i}(x)=G_{i}(x)-\frac{1}{3} B_{i}(x)$
$N_{i}(x)=\frac{2}{3} B_{i}(x)-H_{i}(x)$

$N_{i}(x)=\frac{2}{3} G_{i}(x)-\frac{1}{3} H_{i}(x)$

Using (10), (13) and (19), we obtain the following integral representation of $N_{i}(x)$ :

$N_{i}(x)=\frac{2}{3 \pi} \int_{0}^{\infty} \sin \left(x t+\frac{1}{3} t^{3}\right) d t-$ $\frac{1}{3 \pi} \int_{0}^{\infty} \exp \left(x t-\frac{1}{3} t^{3}\right) d t$

First and second derivatives of $N_{i}(x)$ are given by $\frac{d N_{i}(x)}{d x}=\frac{d A_{i}(x)}{d x} \int_{0}^{x} B_{i}(t) d t-\frac{d B_{i}(x)}{d x} \int_{0}^{x} A_{i}(t) d t$ (21)

$\frac{d^{2} N_{i}(x)}{d x^{2}}=-W\left(A_{i}(x), B_{i}(x)\right)=\frac{1}{\pi}$

Equations (16) and (21) together with Tables 1(a) and $1(b)$, provide the following values at $x=0$ :

$N_{i}(0)=\frac{d N_{i}(0)}{d x}=0$

which are needed to evaluate the arbitrary constants, $d_{1}$ and $d_{2}$, and write the solution to the initial value problem, (1), (2), (3), with $f(x)=R$, as

$y=\pi\left\{\frac{(3)^{\frac{1}{6}} \alpha}{\Gamma\left(\frac{1}{3}\right)}-\frac{\beta}{(3)^{\frac{1}{6}} \Gamma\left(\frac{2}{3}\right)}\right\} A_{i}(x)+\pi\left\{\frac{\alpha}{(3)^{\frac{1}{3}} \Gamma\left(\frac{1}{3}\right)}+\right.$

$\left.\frac{\beta}{(3)^{\frac{2}{3}} \Gamma\left(\frac{2}{3}\right)}\right\} B_{i}(x)-\pi R N_{i}(x)$

Detailed solutions, and computations, to initial and boundary value problems when the forcing function is constant are provided in reference [23].

\section{Remark 1.}

We remark at the outset that in solving the initial value problem (1), (2), (3), and expressing the solution in terms of $N_{i}(x)$, values of the arbitrary 
constants are independent of the forcing function. To illustrate this point further, when $f(x)=\mp \frac{1}{\pi}$, solution to the initial value problem is simply given by equation (24) with $R=\mp \frac{1}{\pi}$, while coefficients of Airy's functions (values of the arbitrary constants) in the solution do not change. By comparison, when the general solution is expressed in terms of Scorer functions, $G_{i}(x)$ and $H_{i}(x)$, coefficients of Airy's functions do in fact change.

\section{A. 4. The Case of Variable Forcing Function}

When the forcing function is any differentiable function $f(x)$, the complementary solution is a linear combination of Airy's functions, and takes the form $y_{c}=e_{1} A_{i}(x)+e_{2} B_{i}(x)$, where $e_{1}$ and $e_{2}$ are arbitrary constants. Particular solution, $y_{p}$, of (1) can be obtained using variations of parameters by letting $y_{p}=v_{1} A_{i}(x)+v_{2} B_{i}(x)$. Computing $v_{1}$ and $v_{2}$ yields, after some simplification, $y_{p}=\pi K_{i}(x)-$ $\pi f(x) N_{i}(x)$, and the general solution to (1) thus takes the form $y=y_{c}+y_{p}$, or

$y=e_{1} A_{i}(x)+e_{2} B_{i}(x)+\pi K_{i}(x)-\pi f(x) N_{i}(x)$

where the function $K_{i}(x)$, referred to as the Standard Nield-Kuznetsov Function of the Second Kind, takes the form

$$
\begin{aligned}
& K_{i}(x)=A_{i}(x) \int_{0}^{x}\left\{\int_{0}^{t} B_{i}(\tau) d \tau\right\} f^{\prime}(t) d t- \\
& B_{i}(x) \int_{0}^{x}\left\{\int_{0}^{t} A_{i}(\tau) d \tau\right\} f^{\prime}(t)
\end{aligned}
$$

and $f^{\prime}$ denotes the derivative of $f$ with respect to its argument.

Equation (26) can be written in the following form in order to establish the connection between $K i(x)$ and $N i(x)$

$$
\begin{aligned}
& K_{i}(x)=f(x) N_{i}(x)-\left\{A_{i}(x) \int_{0}^{x} f(t) B_{i}(t) d t-\right. \\
& \left.B_{i}(x) \int_{0}^{x} f(t) A_{i}(t) d t\right\}
\end{aligned}
$$

The following first derivative of $K i(x)$ is obtained from (27):

$$
\begin{aligned}
& \frac{d K_{i}(x)}{d x}=f^{\prime}(x) N_{i}(x)+f(x) \frac{d N_{i}(x)}{d x}- \\
& \left\{\frac{d A_{i}(x)}{d x} \int_{0}^{x} f(t) B_{i}(t) d t-\frac{d B_{i}(x)}{d x} \int_{0}^{x} f(t) A_{i}(t) d t\right\}
\end{aligned}
$$

Equations (23), (27) and (28) furnish the values for $K_{i}(0)$ and $\frac{d K_{i}(0)}{d x}$ :

$K_{i}(0)=\frac{d K_{i}(0)}{d x}=0$

Using conditions (2) and (3), we verify that the values of arbitrary constants $e_{1}$ and $e_{2}$ are the same as $d_{1}$ and $d_{2}$, respectively, and the solution to the initial value problem (1), (2) and (3), when $f(x)$ is a variable function of $x$, is given by

$$
\begin{aligned}
& y=\pi\left\{\frac{(3)^{\frac{1}{6}} \alpha}{\Gamma\left(\frac{1}{3}\right)}-\frac{\beta}{(3)^{\frac{1}{6}} \Gamma\left(\frac{2}{3}\right)}\right\} A_{i}(x)+\pi\left\{\frac{\alpha}{(3)^{\frac{1}{3}} \Gamma\left(\frac{1}{3}\right)}+\right. \\
& \left.\frac{\beta}{(3)^{\frac{2}{3}} \Gamma\left(\frac{2}{3}\right)}\right\} B_{i}(x)+\pi K_{i}(x)-\pi f(x) N_{i}(x)
\end{aligned}
$$

\section{Remark 2.}

The function $N_{i}(x)$, defined in equation (16), is referred to as the Standard Nield-Kuznetsov Function of the First Kind. Particular solution to the inhomogeneous Airy's ODE with a constant forcing function is expressed in terms of $N_{i}(x)$. This function was introduced by Nield and Kuznetsov, [18]. Its extensive properties and relationship to other functions, such as Bessel functions, were studied in details and established in the work of Hamdan and Kamel, [21].

The function $K_{i}(x)$, defined in equation (27), is referred to as the Standard Nield-Kuznetsov Function of the Second Kind. Particular solution to the inhomogeneous Airy's ODE with a variable forcing function is expressed in terms of $K_{i}(x)$. This function was introduced by Hamdan and Kamel, [21]. 


\section{A.5. Computations of the Standard Nield-} Kuznetsov Functions

The standard Nield-Kuznetsov functions of the first and second kinds can be evaluated using asymptotic or ascending series $(c f .[4,18,19,20,22,26,27]$. Ascending series expressions are shown below, [26, 27]:

$N_{i}(x)=$

$2 \sqrt{3} a_{1} a_{2}\left[\left\{\sum_{k=0}^{\infty}\left(\frac{1}{3}\right)_{k} \frac{3^{k} x^{3 k}}{(3 k) !}\right\}\left\{\sum_{k=0}^{\infty}\left(\frac{2}{3}\right)_{k} \frac{3^{k} x^{3 k+2}}{(3 k+2) !}\right\}-\right.$ $\left.\left\{\sum_{k=0}^{\infty}\left(\frac{1}{3}\right)_{k} \frac{3^{k} x^{3 k+1}}{(3 k+1) !}\right\}\left\{\sum_{k=0}^{\infty}\left(\frac{2}{3}\right)_{k} \frac{3^{k} x^{3 k+1}}{(3 k+1) !}\right\}\right]$

$K_{i}(x)=2 \sqrt{3} a_{1} a_{2} *$

$$
\left[\begin{array}{c}
\left\{\sum_{k=0}^{\infty}\left(\frac{1}{3}\right)_{k} \frac{3^{k} x^{3 k}}{(3 k) !}\right\}\left\{\sum_{k=0}^{\infty}\left(\frac{2}{3}\right)_{k} \int_{0}^{x} F(t) \frac{3^{k} t^{3 k}}{(3 k) !}\right\}- \\
\left\{\sum_{k=0}^{\infty}\left(\frac{1}{3}\right)_{k} \int_{0}^{x} F(t) \frac{3^{k} t^{3 k-1}}{(3 k-1) !}\right\} * \\
\left\{\sum_{k=0}^{\infty}\left(\frac{2}{3}\right)_{k} \frac{3^{k} x^{3 k+1}}{(3 k+1) !}\right\}
\end{array}\right]
$$

where

$a_{1}=A_{i}(0) \approx 0.3550280538878172$

$a_{1}=-\frac{d}{d x} A_{i}(0) \approx 0.2588194037928067$

$(b)_{k}=\frac{\Gamma(b+k)}{\Gamma(b)}=b(b+1)(b+2) \ldots(b+k-$

$1) ; k>0$

is the Pochhammer symbol, [7], where $(b)_{0}=1$.

Extensive tabulations have been carried out by Alzahrani et.al. [28, 29, 30, 31]. In Tables 2(a) and 2(b), below, are some values of $K_{i}(x)$ and $N_{i}(x)$ at selected values of $x$. Results were obtained using ascending series truncated after 10 terms.

\begin{tabular}{|l|l|l|}
\hline$F(x)$ & $K_{i}(x=0.5)$ & $K_{i}(x=1)$ \\
\hline
\end{tabular}

\begin{tabular}{|c|c|c|}
\hline$x$ & -0.04003797109116 & -0.16725609184051 \\
\hline$x^{2}$ & -0.01331827008945 & -0.10968956771722 \\
\hline $\sin x$ & -0.03921345759006 & -0.15411426990974 \\
\hline$e^{x}$ & -0.20843678166552 & -0.58437891995633 \\
\hline
\end{tabular}

Table 2(a)

\begin{tabular}{|c|c|}
\hline $\boldsymbol{N}_{\boldsymbol{i}}(\boldsymbol{x}=\mathbf{0 . 1})$ & -0.0015916299 \\
\hline $\boldsymbol{N}_{\boldsymbol{i}}(\boldsymbol{x}=\mathbf{0 . 5})$ & -0.04003797119 \\
\hline $\boldsymbol{N}_{\boldsymbol{i}}(\boldsymbol{x}=\mathbf{1})$ & -0.1672560919 \\
\hline
\end{tabular}

Table 2(b)

\section{ThE GENERALIZED NiELD-KuZNETSOV FUNCTIONS}

\section{A. Solution to the Homogeneous Generalized Airy's Equation}

Consider the generalized inhomogeneous Airy's ODE of the form:

$\frac{d^{2} y}{d x^{2}}-x^{n} y=f(x)$

In their elegant analysis, Swanson and Headley, [9], provided solution to the homogeneous part of (33) as a linear combination of the linearly independent functions $A_{n}(x)$, and $B_{n}(\mathrm{x})$, referred to as the generalized Airy's functions of the first and second kinds, respectively, namely:

$y=a_{1} A_{n}(x)+a_{2} B_{n}(x)$

where $a_{1}$ and $a_{2}$ are arbitrary constants and

$A_{n}(x)=\frac{2 p}{\pi} \sin (p \pi)(x)^{\frac{1}{2}} K_{p}(\zeta)$

$B_{n}(x)=(p x)^{\frac{1}{2}}\left(I_{-p}(\zeta)+I_{p}(\zeta)\right.$

(36)

The terms $I_{p}$ and $K_{p}$ are the modified Bessel functions defined as:

$I_{p}(\zeta)=i^{-p} J_{p}(i \zeta)=\sum_{m=1}^{\infty} \frac{1}{m ! \Gamma(m+p+1)}\left(\frac{\zeta}{2}\right)^{2 m+p}$ 


$$
K_{p}(\zeta)=\frac{\pi}{2} \frac{\left(I_{-p}(\zeta)-I_{p}(\zeta)\right)}{\sin (p \pi)}
$$

with $p=\frac{1}{n+2}, \zeta=2 p x$, and $\Gamma($.$) is the gamma$ function.

The Wronskian of $A_{n}(x)$ and $B_{n}(x)$ is given by:

$$
W\left(A_{n}(x), B_{n}(x)\right)=\frac{2}{\pi} p^{\frac{1}{2}} \sin (p \pi)
$$

and the first derivatives of the generalized Airy's functions are given by, [9]:

$\frac{d A_{n}(x)}{d x}=-\frac{2 p}{\pi} \sin (p \pi)(x)^{\frac{n+1}{2}} K_{p-1}(\zeta)$

$\frac{d B_{n}(x)}{d x}=p^{\frac{1}{2}}(x)^{\frac{n+1}{2}}\left[I_{1-p}(\zeta)+I_{p-1}(\zeta)\right]$ $(41)$

Swanson and Headley [9] extensively studied properties of the generalized Airy's functions, $A_{n}(x)$, and $B_{n}(\mathrm{x})$, and their connections to other functions, such as Bessel functions.

In their modelling of flow through variable permeability porous layers, Abu Zaytoon et.al., [19], showed that the flow equations could be reduced to the inhomogeneous Airy's ODE (33). In order to obtain solutions to initial value problems or boundary value problems, they solved (33) using variations of parameters and obtained the following cases of solutions.

\section{B. The Case of Constant Forcing Function}

When $f(x)=R=$ constant, Abu Zaytoon et.al., [19], showed that the general solution to (33) takes the form

$y_{n}=b_{1} A_{n}(x)+b_{2} B_{n}(x)-\frac{\pi R}{2 \sqrt{p} \sin (p \pi)} N_{n}(x)$ where $b_{1}$ and $b_{2}$ are arbitrary constants, and

$N_{n}(x)=A_{n}(x) \int_{0}^{x} B_{n}(t) d t-B_{n}(x) \int_{0}^{x} A_{n}(t) d t$

with first derivative given by

$$
\frac{d N_{n}(x)}{d x}=\frac{d A_{n}(x)}{d x} \int_{0}^{x} B_{n}(t) d t-\frac{d B_{n}(x)}{d x} \int_{0}^{x} A_{n}(t) d t
$$

\section{Remark 3.}

Integral function (43) is termed the Generalized Nield-Kuznetsov Function of the First Kind, as introduced by Abu Zaytoon et.al. [19]. Clearly, when $n=1$, this function reduces to the Standard Nield-Kuznetsov Function of the First Kind, equation (16).

\section{The Case of Variable Forcing Function}

When the forcing function, $f(x)$, is a variable function of $x$, solution to (33) takes the form, [29]:

$y_{n}=c_{1} A_{n}(x)+c_{2} B_{n}(x)-\frac{\pi}{2 \sqrt{p} \sin (p \pi)} K_{n}(x)$

where $c_{1}$ and $c_{2}$ are arbitrary constants, and

$K_{n}(x)=B_{n}(x) \int_{0}^{x} F(t) \frac{d A_{n}(t)}{d t} d t-$ $A_{n}(x) \int_{0}^{x} F(t) \frac{d B_{n}(t)}{d t} d t$

The function $K_{n}(x)$ is referred to as the Generalized Nield-Kuznetsov Function of the Second Kind. Its first derivative is given by, [29]:

$$
\begin{aligned}
& \frac{d K_{n}(x)}{d x}=\frac{d B_{n}(x)}{d x} \int_{0}^{x} F(t) \frac{d A_{n}(t)}{d t} d t- \\
& \frac{d A_{n}(x)}{d x} \int_{0}^{x} F(t) \frac{d B_{n}(t)}{d t} d t-\frac{2 \sqrt{p} \sin (p \pi)}{\pi} F(x)
\end{aligned}
$$

wherein $F^{\prime} \equiv f$. 
D. Computations of the Standard Nield-Kuznetsov Functions

Following Swanson and Headley, [9], the generalized Airy's functions are evaluated as follows. Letting

$p=\frac{1}{n+2}, \alpha_{n}=\frac{(p)^{1-p}}{\Gamma(1-p)}, \beta_{n}=\frac{(p)^{p}}{\Gamma(p)}$

$g_{n 1}(x)=1+\sum_{k=1}^{\infty} p^{2 k} \prod_{j=1}^{k} \frac{x^{(n+2) k}}{j(j-p)}$

$g_{n 2}(x)=1+\sum_{k=1}^{\infty} p^{2 k} \prod_{j=1}^{k} \frac{x^{(n+2) k}}{j(j-p)}$

$(50)$

then

$A_{n}(x)=\alpha_{n} g_{n 1}(x)-\beta_{n} g_{n 2}(x)$

$(51)$

$B_{n}(x)=\frac{1}{\sqrt{p}}\left[\alpha_{n} g_{n 1}(x)+\beta_{n} g_{n 2}(x)\right.$

The generalized Nield-Kuznetsov functions can be evaluated using (41)-(44) and the following expressions, [19, 29, 30, 31]:

$N_{n}(x)=\frac{2}{\sqrt{p}} \alpha_{n} \beta_{n}\left\{g_{n 1}(x) \int_{0}^{x} g_{n 2}(t) d t-\right.$

$\left.g_{n 2}(x) \int_{0}^{x} g_{n 1}(t) d t\right\}$

$K_{n}(x)=\frac{-2}{\sqrt{p}} \alpha_{n} \beta_{n}\left\{g_{n 1}(x) \int_{0}^{x} F(t) \frac{d}{d t} g_{n 2}(t) d t-\right.$ $\left.g_{n 2}(x) \int_{0}^{x} F(t) \frac{d}{d t} g_{n 1}(t) d t\right\}$

(54)

Upon evaluating (51), (52), (43), (44), (46), and (47) at $x=0$, we obtain the following values in Table 3 ..

Computations of the generalized Nield-Kuznetsov functions, using (53) and (54), have been carried out and tabulated by Alzahrani et.al. [30], for $\mathrm{n}=1,2, \ldots, 10$. It is worth noting that (54) reduces to
(53) when $F(x)=x$, and $x=1$, as verified by values of $N_{n}(1)$ and $K_{n}(1)$ in Tables 4 and 5 below.

\begin{tabular}{l}
$\begin{array}{l}\text { Values of Generalized Airy's } \\
\text { and Nield-Kuznetsov } \\
\text { Functions, and Derivatives } \\
\text { at Zero }\end{array}$ \\
An $(0)=\frac{(p)^{1-p}}{\Gamma(1-p)}$ \\
\hline$B_{n}(0)=\frac{(p)^{1 / 2-p}}{\Gamma(1-p)}$ \\
\hline$\frac{d A_{n}(0)}{d x}=-\frac{(p)^{p}}{\Gamma(p)}$ \\
\hline$\frac{d B_{n}(0)}{d x}=-\frac{(p)^{p-1 / 2}}{\Gamma(p)}$ \\
\hline$N_{n}(0)=0$ \\
$\frac{d N_{n}(0)}{d x}=0$ \\
\hline$K_{n}(0)=0$ \\
$\frac{d K_{n}(0)}{d x}$ \\
$=-\frac{2 \sqrt{p} \sin (p \pi)}{\pi} F(0)$ \\
\hline
\end{tabular}

Table 3

Values of $N_{n}(1)$ for $n=$ $1,2,3,4,5$, and 10

$N_{1}(1)=-0.1671679498$
$N_{2}(1)=-0.1163218192$
$N_{3}(1)=-0.08567790804$
$N_{4}(1)=-0.06614082173$
$N_{5}(1)=-0.05292832891$




$$
N_{10}(1)=-0.02391327336
$$

\section{Table 4}

\begin{tabular}{|l|c|c|}
\hline $\begin{array}{l}\text { Values } \\
\text { of } \\
\boldsymbol{K}_{\boldsymbol{n}}(\mathbf{1})\end{array}$ & $\boldsymbol{F}(\boldsymbol{x})=\boldsymbol{x}$ & $\boldsymbol{F}(\boldsymbol{x})=\boldsymbol{x}^{\mathbf{2}}$ \\
\hline$K_{n}(1)$ & $F(x)=x$ & $F(x)=x^{2}$ \\
\hline$K_{1}(1)$ & -0.1671679498 & -0.1095475388 \\
\hline$K_{2}(1)$ & -0.1163218194 & -0.07681056585 \\
\hline$K_{3}(1)$ & -0.08567790816 & -0.05678057772 \\
\hline$K_{4}(1)$ & -0.06614082166 & -0.04391997436 \\
\hline$K_{5}(1)$ & -0.05292832894 & -0.03518808431 \\
\hline$K_{10}(1)$ & -0.02391327336 & -0.01593053627 \\
\hline
\end{tabular}

Table 5

\section{THE PARAMETRIC NIELD-KUZNETSOV FUNCTIONS}

\section{A. Weber's Homogeneous Equation}

Weber's inhomogeneous differential equation finds applications in the study of fluid flow through porous layers with variable permeability, [20]. Weber's homogeneous equation takes the form $\frac{d^{2} y}{d x^{2}}+\left(a x^{2}+b x+c\right) y=0$ (55)

where $a, b, c$ are constants and the independent variable, $x$, is in general complex.

However, it has been reported in the literature in other forms, ( $c f .[1,2,3])$ that include three distinctive forms whose mathematical and physical applications have been discussed in the work of Temme, [2]. Of interest, due to its validity in the real domain and its usefulness in modelling practical situations in the real plain, is Weber's equation of the form

$\frac{d^{2} y}{d x^{2}}+\left(\frac{x^{2}}{4}-a\right) y=0$

(56)
Equation (56) possesses the solutions $W(a, \mp x)$, where the variable $x$ and the parameter $a$ are real numbers. These solutions represent a linearly independent, numerically satisfactory pair of solutions, [2], for all $x \in \mathfrak{R}$, with the Wronskian given by

$$
w(W(a, x), W(a,-x))=1
$$

Abu Zaytoon et.al. [20] encountered applications in the study of flow through porous layers, of the inhomogeneous Weber's equation, namely:

$\frac{d^{2} y}{d x^{2}}+\left(\frac{x^{2}}{4}-a\right) y=f(x)$

(58)

and showed that a particular solution is given by

$y_{p}=W(a,-x) \int_{0}^{x} f(t) W(a, t) d t-$

$W(a, x) \int_{0}^{x} f(t) W(a,-t) d t$

The following two cases arise, depending on $f(x)$.

B. The Case of $f(x)=R=$ constant

If $f(x)=R$, then

$y_{p}=R\left\{W(a,-x) \int_{0}^{x} f(t) W(a, t) d t-\right.$

$\left.W(a, x) \int_{0}^{x} f(t) W(a,-t) d t\right\}$

In equation (60), the expression in brackets is termed the Parametric Nield-Kuznetsov Function of the First Kind, which depends on parameter $a$, and is denoted and given by

$N_{w}(a, x)=W(a, x) \int_{0}^{x} W(a,-t) d t-$ $W(a,-x) \int_{0}^{x} W(a, t) d t$

with a first derivative given by

$\frac{d N_{W}(a, x)}{d x}=\frac{d W(a, x)}{d x} \int_{0}^{x} W(a,-t) d t+$ $\frac{d W(a,-x)}{d x} \int_{0}^{x} W(a, t) d t$ 
General solution to (58) can thus be written as:

$y=c_{1} W(a, x)+c_{2} W(a,-x)-R N_{w}(a, x)$

where $c_{1}$ and $c_{2}$ are arbitrary constants.

C. The Case of $f(x)$ Being a Variable Function of $x$

If $f(x)$ is a variable function of $x$, then

$y_{p}=W(a,-x) \int_{0}^{x} F^{\prime}(t) W(a, t) d t-$

$W(a, x) \int_{0}^{x} F^{\prime}(t) W(a,-t) d t$

(64)

where $F^{\prime}(t)=f(t)$.

Using integration by parts, equation (64) can be written as:

$y_{p}=-\left\{W(a,-x) \int_{0}^{x} F(t) \frac{d W(a, t)}{d t} d t+\right.$

$\left.W(a, x) \int_{0}^{x} F(t) \frac{d W(a,-t)}{d t} d t\right\}$

The expression on the right-hand-side of (53) depends on parameter $a$, and is reminiscent of the Nield-Kuznetsov function of the second kind. It is referred to as the Parametric Nield-Kuznetsov Function of the Second Kind, denoted by $K_{w}(a, x)$, and defined as:

$K_{w}(a, x)=W(a,-x) \int_{0}^{x} F(t) \frac{d W(a, t)}{d t} d t+$

$W(a, x) \int_{0}^{x} F(t) \frac{d W(a,-t)}{d t} d t$

(66)

with first derivative given by

$\frac{d K_{W}(a, x)}{d x}=\frac{d W(a, x)}{d x} \int_{0}^{x} F(t) \frac{d W(a,-t)}{d t} d t-$ $\frac{d W(a,-x)}{d x} \int_{0}^{x} F(t) \frac{d W(a, t)}{d t} d t-F(x)$

(67)
General solution to (58) can thus be written as:

$y=d_{1} W(a, x)+d_{2} W(a,-x)-K_{w}(a, x)$

where $d_{1}$ and $d_{2}$ are arbitrary constants.

In solving initial and boundary value problems involving the Weber ODE, one requires values of the parametric Nield-Kuznetsov functions at given values of the argument. Values at $x=0$ of $W(a, x)$, $\frac{d W}{d x}(a, x)$ are given by, $[14,15,17]$ :

$W(a, 0)=\frac{1}{(2)^{\frac{3}{4}}}\left|\frac{\Gamma\left(\frac{1}{4}+\frac{1}{2} i a\right)}{\Gamma\left(\frac{3}{4}+\frac{1}{2} i a\right)}\right|^{\frac{1}{2}}$

$W(0,0)=\frac{1}{(2)^{\frac{3}{4}}}\left|\frac{\Gamma\left(\frac{1}{4}\right)}{\Gamma\left(\frac{3}{4}\right)}\right|^{\frac{1}{2}}$

(70)

$\frac{d W}{d x}(a, 0)=-\frac{1}{(2)^{\frac{1}{4}}}\left|\frac{\Gamma\left(\frac{3}{4}+\frac{1}{2} i a\right)}{\Gamma\left(\frac{1}{4}+\frac{1}{2} i a\right)}\right|^{\frac{1}{2}}$

$\frac{d W}{d x}(a, 0)=-\frac{1}{(2)^{\frac{1}{4}}}\left|\frac{\Gamma\left(\frac{3}{4}\right)}{\Gamma\left(\frac{1}{4}\right)}\right|^{\frac{1}{2}}$

Values of the parametric Nield-Kuznetsov functions and derivatives at $x=0$ are provided in Table 6 , below.

\begin{tabular}{|c|l|}
\hline $\begin{array}{l}\text { Parametric } \\
\text { Nield-Kuznetsov } \\
\text { of first kind and } \\
\text { derivative at } \\
\text { zero }\end{array}$ & $\begin{array}{l}\text { Parametric Nield- } \\
\text { Kuznetsov of second } \\
\text { kind and derivative } \\
\text { at zero }\end{array}$ \\
\hline$N_{w}(a, 0)=0$ & $K_{w}(a, 0)=0$ \\
$\frac{d N_{w}}{d x}(a, 0)=0$ & $\frac{d K_{w}}{d x}(a, 0)=-F(0)$ \\
\hline
\end{tabular}




\section{Table 6}

D. Computations of the Parametric Nield-Kuznetsov Functions

The following expressions, developed in [13-17], are used in the computations of the parabolic cylindrical functions $W(a, x)$ and $W(a,-x)$ :

$W(a, x)=W(a, 0) \sum_{n=0}^{\infty} \rho_{n}(a) \frac{x^{2 n}}{(2 n) !}+$ $\frac{d W}{d x}(a, 0) \sum_{n=0}^{\infty} \delta_{n}(a) \frac{x^{2 n+1}}{(2 n+1) !}$

(73)

$W(a,-x)=W(a, 0) \sum_{n=0}^{\infty} \rho_{n}(a) \frac{x^{2 n}}{(2 n) !}-$ $\frac{d W}{d x}(a, 0) \sum_{n=0}^{\infty} \delta_{n}(a) \frac{x^{2 n+1}}{(2 n+1) !}$

(74)

$\frac{d}{d x} W(a, x)=W(a, 0) \sum_{n=0}^{\infty} \rho_{n}(a) \frac{x^{2 n-1}}{(2 n-1) !}+$ $\frac{d W}{d x}(a, 0) \sum_{n=0}^{\infty} \delta_{n}(a) \frac{x^{2 n}}{(2 n) !}$

(75)

$\frac{d}{d x} W(a,-x)=-W(a, 0) \sum_{n=0}^{\infty} \rho_{n}(a) \frac{x^{2 n-1}}{(2 n-1) !}+$ $\frac{d W}{d x}(a, 0) \sum_{n=0}^{\infty} \delta_{n}(a) \frac{x^{2 n}}{(2 n) !}$

(76)

Using (73)-(76), Alzahrani et al [23, 28], developed the following series expressions for the parametric Nield-Kuznitsov functions:

$N_{w}(a, x)=2 W(a, 0) \frac{d W}{d x}(a, 0)^{*}$

$\left[\left\{\sum_{n=0}^{\infty} \delta_{n}(a) \frac{x^{2 n+1}}{(2 n+1) !}\right\}\left\{\sum_{n=0}^{\infty} \rho_{n}(a) \frac{x^{2 n+1}}{(2 n+1) !}\right\}-\right.$ $\left.\left\{\sum_{n=0}^{\infty} \rho_{n}(a) \frac{x^{2 n}}{(2 n) !}\right\}\left\{\sum_{n=0}^{\infty} \delta_{n}(a) \frac{x^{2 n+2}}{(2 n+2) !}\right\}\right]$

$K_{w}(a, x)=2 W(a, 0) \frac{d W}{d x}(a, 0) *$

$\left[\sum_{n=0}^{\infty} \rho_{n}(a) \frac{x^{2 n}}{(2 n) !}\right] \int_{0}^{x} \delta_{n}(a) F(t) \frac{t^{2 n}}{(2 n) !} d t$

(78) where

$\rho_{n+2}=a \rho_{n+1}-\frac{1}{2}(n+1)(2 n+1) \rho_{n}$

(79)

$\delta_{n+2}=a \delta_{n+1}-\frac{1}{2}(n+1)(2 n+3) \delta_{n}$

$\rho_{0}(a)=\delta_{0}(a)=1$

$\rho_{1}(a)=\delta_{1}(a)=a$

Sample calculations of $N_{w}(a, x)$ and $K_{w}(a, x)$ using the (77)-(82) are shown in Tables 7 , 8 and 9 , below.

Further computations and solutions to initial and boundary value problems involving the six NieldKuznetsov functions have been carried out by Alzahrani et al [23, 28].

\begin{tabular}{|l|l|l|}
\hline $\boldsymbol{x}$ & $\begin{array}{l}\text { Some Values of } \\
N_{w}(a, x) \\
\boldsymbol{N}_{\boldsymbol{w}}(\boldsymbol{a}, \boldsymbol{x}) ; \boldsymbol{a}=\mathbf{0}\end{array}$ & $\begin{array}{l}\text { Some Values of } \\
N_{w}(a, x)\end{array}$ \\
\hline & $\boldsymbol{N}, \boldsymbol{x}) ; \boldsymbol{a}=\mathbf{1}$ \\
\hline 0 & 0 & 0 \\
\hline 0.1 & -0.004999995827 & -0.0050041638 \\
\hline 0.2 & -0.01999973332 & -0.02006648827 \\
\hline 0.3 & -0.04499696254 & -0.0453354595 \\
\hline 0.4 & -0.07998293445 & -0.08105513505 \\
\hline
\end{tabular}


INTERNATIONAL JOURNAL OF CIRCUITS, SYSTEMS AND SIGNAL PROCESSING

\begin{tabular}{|l|l|l|}
\hline 0.5 & -0.1249349069 & -0.1275598486 \\
\hline 0.6 & -0.1798056698 & -0.1852664744 \\
\hline 0.7 & -0.2445101224 & -0.2546639319 \\
\hline 0.8 & -0.3189089751 & -0.3362995397 \\
\hline 0.9 & -0.4027896942 & -0.4307617641 \\
\hline 1 & -0.4958448910 & -0.5386588510 \\
\hline
\end{tabular}

Table 7

\begin{tabular}{|c|c|c|}
\hline$x$ & $\begin{array}{c}\boldsymbol{K}_{\boldsymbol{w}}(\boldsymbol{a}, \boldsymbol{x}) ; \boldsymbol{a}=\mathbf{0} \\
F(x)=x^{2}\end{array}$ & $\begin{array}{c}\boldsymbol{K}_{\boldsymbol{w}}(\boldsymbol{a}, \boldsymbol{x}) ; \boldsymbol{a}=\mathbf{1} \\
F(x)=x^{2}\end{array}$ \\
\hline 0 & 0 & 0 \\
\hline 0.1 & -0.000333331745 & -0.000336005395 \\
\hline 0.2 & -0.002666463495 & -0.002752690054 \\
\hline 0.3 & -0.008996529010 & -0.009659773517 \\
\hline 0.4 & -0.02130733755 & -0.02415201443 \\
\hline 0.5 & -0.04154278194 & -0.05041843662 \\
\hline 0.6 & -0.07155657245 & -0.09422931145 \\
\hline 0.7 & -0.1130311074 & -0.1635219131 \\
\hline 0.8 & -0.1673594862 & -0.2690996788 \\
\hline
\end{tabular}

\begin{tabular}{|l|l|l|}
\hline 0.9 & -0.2354868836 & -0.4254486950 \\
\hline 1 & -0.3177111593 & -0.6516586206 \\
\hline
\end{tabular}

Table 8

\begin{tabular}{|c|c|c|}
\hline$y$ & $\begin{array}{c}\boldsymbol{K}_{\boldsymbol{w}}(\boldsymbol{a}, \boldsymbol{x}) ; \boldsymbol{a}=\mathbf{0} \\
F(x)=\sin x\end{array}$ & $\begin{array}{c}\boldsymbol{K}_{\boldsymbol{w}}(\boldsymbol{a}, \boldsymbol{x}) ; \boldsymbol{a}=\mathbf{1} \\
F(x)=\sin x\end{array}$ \\
\hline 0 & 0 & 0 \\
\hline 0.1 & -0.004995813904 & -0.005033369365 \\
\hline 0.2 & -0.01993209438 & -0.02053562388 \\
\hline 0.3 & -0.04464846700 & -0.04772579586 \\
\hline 0.4 & -0.07885511745 & -0.08867593176 \\
\hline 0.5 & -0.1221006021 & -0.1463657018 \\
\hline 0.6 & -0.1737300984 & -0.2247467511 \\
\hline 0.7 & -0.2328376690 & -0.3288039768 \\
\hline 0.8 & -0.2982174230 & -0.4645964625 \\
\hline 0.9 & -0.3683198455 & -0.6392561548 \\
\hline 1 & -0.4412208948 & -0.8609179616 \\
\hline
\end{tabular}

Table 9 


\section{CONCLUSION}

In this work, we provided a complete overview of the recent developments and advances over the past decade of the three classes of the Nield-Kuznetsov functions. The most important, and novel aspect of this work is that it provides the basis for solutions to the general, inhomogeneous Airy's and Weber's equations with either constant or variable forcing functions. Many problems in Mathematical Physics that are modelled using these equations can now be easily handled with the introduced functions.

The Standard Nield-Kuznetsov functions of the first and second kinds arise due to the solution of Airy's inhomogeneous ODE with constant and variable forcing functions, respectively. They are defined by equations (16) and (27), respectively, and their ascending series expressions are given by equations (31) and (32), respectively.

The Generalized Nield-Kuznetsov functions of the first and second kinds arise due to the solution of generalized Airy's inhomogeneous ODE with constant and variable forcing functions, respectively. They are defined by equations (53) and (54), respectively, and their series expressions are given by equations (31) and (32), respectively.

The Parametric Nield-Kuznetsov functions of the first and second kinds arise due to the solution of Weber's inhomogeneous ODE with constant and variable forcing functions, respectively. They are defined by equations (61) and (66), respectively, and their series expressions are given by equations (77) and (78), respectively.

With this knowledge, one is now able to solve the inhomogeneous Airy's ODE, inhomogeneous generalized Airy's ODE and inhomogeneous Weber's ODE that involve initial value problems and two-point boundary value problems.

Evaluation of all of the Nield-Kuznetsov functions relies heavily on computer simulation.
While Airy's functions and their series representations have built-in definitions in Maple and Mathematica, the Nield-Kuznetsov functions are not presently included. Series presentations for the Nield-Kuznetsov functions are available and can be evaluated using Maple or Mathematica, as has been done in [19] to [31]. For the parametric NieldKuznetsov functions, the most advanced and stateof-the-art computational methods found in references [13] to [20] have been used in their evaluations.

\section{REFERENCES:}

[1] M. Abramowitz and I.A. Stegun, Handbook of Mathematical Functions, Dover, New York 1984.

[2] N. M., Temme, "Parabolic Cylinder Functions", in NIST Handbook of Mathematical Functions", Chapter 12, pp. 303319, (Frank W. J. Olver, Daniel W. Lozier, Ronald F. Boisvert and Charles W. Clark, eds.), U.S. Dept. Commerce, Washington, DC, Cambridge University, 2000.

[3] N.M. Temme. Special functions: An introduction to the classical functions of mathematical physics. John Wiley \& Sons, New York, 1996.

[4] O. Vallée and M. Soares, Airy functions and applications to physics. World Scientific, London, 2004.

[5] J. C. P. Miller and Z. Mursi, Notes on the solution of the equation $y^{\prime \prime}-x y=f(x)$, Quarterly J. Mech. Appl. Math., vol. 3, pp. 113$118,1950$.

[6] R.S. Scorer, "Numerical evaluation of integrals of the form $I=\int_{x 1}^{x} f(x) e^{l \varphi(x)} d x_{0}$ and the $-u^{3} d u$ ", Quarterly J. Mech. Applo Math., vol. 3, pp. 107-112, 1950.

[7] S.-Y. Lee, "The inhomogeneous Airy functions, Gi(z) and Hi(z)", J. Chem. Phys. vol. 72, pp. 332-336, 1980.

[8] T.M. Dunster, "Uniform asymptotic expansions for solutions of the parabolic cylinder and Weber equations", J. Classical Analysis, vol. 17, No. 1, pp. 69-107, 2021.

[9] C.A. Swanson and V.B. Headley, "An extension of Airy's equation". SIAM Journal of Applied Mathematics, vol. 15(6), pp. 1400-1412, 1967.

[10] R.M. Corless, D.J. Jeffrey and H. Rasmussen, "Numerical evaluation of Airy functions with complex arguments". J. Comput. Phys. vol. 99, pp. 106-114, 1992.

[11] A.J. MacLeod, Computation of inhomogeneous Airy functions, J. Comput. Appl. Math. vol. 53, pp. 109-116, 1994.

[12] Z. Schulten, R.G. Gordon and D. G. M. Anderson, "A numerical algorithm for the evaluation of Weber parabolic cylinder functions $U(a, x), V(a, x)$, and $W(a,+/-x), ", J$. Comput. Phys. vol. 42(2), pp. 213-217, 1981.'

[13] A. Gil, J. Segura, N.M. Temme, On NonOscillating Integrals for Computing 
inhomogeneous Airy Functions, Mathematics of Computation, vol. 70, pp. 1183-1194, 2001.

[14] A.Gil, J. Segura and N.M. Temme, "Fast and a curate computation of the Weber parabolic cylinder function, IMA Journal of numerical analysis, vol. 31, pp. 1194-1216, 2011.

[15] J. Segura and A. Gil, "Parabolic cylinder functions of integer and half- integer orders of non-negative arguments", Computer Physics Communications, vol. 115, pp. 69-86, 1998.

[16] N.M. Temme, Special Functions: An Introduction to the Classical Functions of Mathematical Physics, Wiley, New York, 1996.

[17] N.M. Temme, "Numerical and asymptotic aspects of parabolic cylinder functions", J. Computational and Applied Mathematics, vol. 121 , pp. 221-246, 2000.

[18] D.A. Nield and A.V. Kuznetsov, "The effect of a transition layer between a fluid and a porous medium: shear flow in a channel", Transport in Porous Media, vol. 78, pp. 477-487, 2009.

[19] M. S. Abu Zaytoon, T. L. Alderson and M. H. Hamdan, "Flow through a layered porous configuration with generalized variable permeability", Int. J. of Enhanced Res. in Sci., Tech. and Eng., vol. 5(6), pp. 1-21, 2016.

[20] M.S. Abu Zaytoon, T.L. Alderson, and M.H. Hamdan, "Weber's inhomogeneous differential equation with initial and boundary conditions", Int. J. Open Problems Compt. Math., vol. 9(2), pp. 1-12, 2016.

[21] M.H. Hamdan and M.T. Kamel, "On the Ni(x) integral function and its application to the Airy's non homogeneous equation", Applied Math. Comput., vol. 21 No. 17, pp. 7349-7360, 2011.

[22] M.H. Hamdan and M.T. Kamel; "A Note on the asymptotic aeries aolution to Airy's inhomogeneous equation", Int. J. Open Problems in Computer Science and Mathematics, vol. 4 (2), pp. 155-161, 2011.

[23] S.M. Alzahrani, I. Gadoura, and M.H. Hamdan, "Initial and boundary value problems involving the inhomogeneous Weber equation and the Nield-Kuznetsov parametric function", The International Journal of Engineering and Science, vol.6(3), pp.9-24, 2017.

[24] M. S. Abu Zaytoon, T. L. Alderson and M. H. Hamdan, "Flow through layered media with embedded transition porous layer", Int. J. of Enhanced Res. in Sci., Tech. and Eng., vol. 5(4), pp. 9-26, 2016.

[25] M. S. Abu Zaytoon, T. L. Alderson and M. H. Hamdan, "Flow through a variable permeability Brinkman porous core", J. Appl. Mathematics and physics, vol. 4, pp. 766- 778, 2016.

[26] S.M. Alzahrani, I. Gadoura and M.H. Hamdan, "Ascending series analysis of the transition layer", Journal of Modern Mechanical Engineering and Technology, vol. 3, pp. 7-16, 2016.

[27] S.M. Alzahrani, I. Gadoura and M.H. Hamdan, "Ascending series solution to Airy's inhomogeneous boundary value problem", Int. J. Open Problems Compt. Math., vol. 9(1), pp. 1-11, 2016.

[28] S.M. Alzahrani, I. Gadoura and M.H. Hamdan, "Representations and computation of the NieldKuznetsov integral iunction", ISOR Journal of Applied Physics, vol. 8(3), pp. 55-67, May-June 2016.

[29] S.M. Alzahrani, I. Gadoura, M.H. Hamdan, "Nield- Kuznetsov functions of the first- and second kind", IOSR Journal of Applied Physics, vol. 8(3), pp. 47-56, May. - Jun. 2016.

[30] S.M. Alzahrani, I. Gadoura, M.H. Hamdan, "Tables of values of the Nield- Kuznetsov functions of the first- and second kind", IOSR Journal of Applied Physics, vol 8(4), Issue 4, pp. 56-67, July-August 2016.

[31] S.M. Alzahrani , M.S. Abu Zaytoon, T.L. Alderson, I. Gadoura and M.H. Hamdan, "On the Nield-Kuznetsov integral function and its applications to Airy's inhomogeneous boundary value problem", Journal of Advances in Physics, vol. 11(5), pp. 3265-3274, January 2016.

[32] M. Himeur, H. Guenfoud and M. Guenfoud, "A Higher order triangular plate finite element using Airy's functions", Advances in Mechanical Engineering, vol. 12(11), pp. 1-19, 2020 .

\section{Contribution of individual authors}

All authors participated in literature review.

S. Jayyousi Dajani analysed Section 2.

M.S. Abu Zaytoon analysed Section 3.

M.H. Hamdan analysed Section 4 and wrote the manuscript.

\section{Sources of funding:}

No funding has been received for this work.

\section{Creative Commons Attribution}

\section{License 4.0 (Attribution 4.0 \\ International , CC BY 4.0)}

This article is published under the terms of the Creative Commons Attribution License 4.0 https://creativecommons.org/licenses/by/4.0/deed.en US 